\title{
On the $x$-coordinates of Pell equations that are sums of two Padovan numbers
}

\section{Mahadi Ddamulira ${ }^{1,2,3}$}

Received: 13 June 2020 / Accepted: 28 September 2020 / Published online: 23 February 2021

(C) The Author(s) 2021

\begin{abstract}
Let $\left(P_{n}\right)_{n \geq 0}$ be the sequence of Padovan numbers defined by $P_{0}=0, P_{1}=P_{2}=1$, and $P_{n+3}=P_{n+1}+P_{n}$ for all $n \geq 0$. In this paper, we find all positive square-free integers $d$ such that the Pell equations $x^{2}-d y^{2}=N$ with $N \in\{ \pm 1, \pm 4\}$, have at least two positive integer solutions $(x, y)$ and $\left(x^{\prime}, y^{\prime}\right)$ such that both $x$ and $x^{\prime}$ are sums of two Padovan numbers.
\end{abstract}

Keywords Padovan number $\cdot$ Pell equation $\cdot$ Linear form in logarithms $\cdot$ Reduction method

Mathematics Subject Classification Primary 11B39 $\cdot$ 11D45 - Secondary $11 \mathrm{D} 61 \cdot 11 \mathrm{~J} 86$

\section{Introduction}

Let $\left(P_{n}\right)_{n \geq 0}$ be the sequence of Padovan numbers defined by the linear recurrence $P_{0}=0, P_{1}=1, P_{2}=1$, and $P_{n+3}=P_{n+1}+P_{n}$ for all $n \geq 0$.

The Padovan sequence appears as sequence $A 000931$ on the On-Line Encyclopedia of Integer Sequences (OEIS) [20]. The first few terms of this sequence are

Supported by the Austrian Science Fund (FWF) projects: F5510-N26-Part of the special research program (SFB), "Quasi-Monte Carlo Methods: Theory and Applications" and W1230_- "Doctoral Program Discrete Mathematics".

$\square$ Mahadi Ddamulira

mddamulira@tugraz.at; mddamulira@mpi-sws.org; mddamulira@cns.mak.ac.ug

1 Institute of Analysis and Number Theory, Graz University of Technology, Kopernikusgasse 24/II, 8010 Graz, Austria

2 Max Planck Institute for Software Systems, Saarland Informatics Campus, Campus E1 5, 66123 Saarbrücken, Germany

3 Department of Mathematics, Makerere University, P.O. Box 7062, Kampala, Uganda 
$0,1,1,1,2,2,3,4,5,7,9,12,16,21,28,37,49,65,86,114,151,200,265,351 \ldots$

Let $d \geq 2$ be a positive square-free integer. It is well known that the Pell equations

$$
x^{2}-d y^{2}= \pm 1
$$

and

$$
X^{2}-d Y^{2}= \pm 4
$$

have infinitely many positive integer solutions $(x, y)$ and $(X, Y)$, respectively. By putting $\left(x_{1}, y_{1}\right)$ and $\left(X_{1}, Y_{1}\right)$ for the smallest positive solutions to (1) and (2), respectively, all the solutions $\left(x_{k}, y_{k}\right)$ and $\left(X_{k}, Y_{k}\right)$ have the form

$$
x_{k}+y_{k} \sqrt{d}=\left(x_{1}+y_{1} \sqrt{d}\right)^{k} \quad \text { for all } k \in \mathbb{Z}^{+},
$$

and

$$
\frac{X_{k}+Y_{k} \sqrt{d}}{2}=\left(\frac{X_{1}+Y_{1} \sqrt{d}}{2}\right)^{k} \quad \text { for all } k \in \mathbb{Z}^{+} \text {. }
$$

Furthermore, $\left(x_{k}\right)_{k \geq 1}$ and $\left(X_{k}\right)_{k \geq 1}$ are binary recurrent sequences. More exactly, the following formulae

$$
x_{k}=\frac{\left(x_{1}+y_{1} \sqrt{d}\right)^{k}+\left(x_{1}-y_{1} \sqrt{d}\right)^{k}}{2},
$$

and

$$
X_{k}=\left(\frac{X_{1}+Y_{1} \sqrt{d}}{2}\right)^{k}+\left(\frac{X_{1}-Y_{1} \sqrt{d}}{2}\right)^{k}
$$

hold for all positive integers $k$.

In the recent years, Luca et al. [17] considered the Diophantine equation

$$
x_{k}=T_{n},
$$

where $x_{k}$ is given by (3) and $\left(T_{n}\right)_{n \geq 0}$ is the Tribonacci sequence defined by $T_{0}=0$, $T_{1}=T_{2}=1$, and $T_{n+3}=T_{n+2}+T_{n+1}+T_{n}$ for all $n \geq 0$. The Tribonacci sequence appears as sequence $A 000073$ on the OEIS [20]. The authors in [17] proved that Eq. (5) has at most one solution $(k, n)$ in positive integers for all $d$ except for $d=2$ when Eq. (5) has the three solutions $(k, n)=\{(1,1),(1,2),(3,5)\}$ and when $d=3$ case in which Eq. (5) has the two solutions $(k, n)=\{(1,3),(2,5)\}$.

Inspired by the main result of Luca et al. [17], E. F. Bravo et al. [3, 4] studied the Diophantine equation

$$
x_{k}=T_{m}+T_{n}
$$

They proved that for each square-free integer $d \geq 2$, there is at most one positive integer $k$ such that $x_{k}$ admits the representation (6) for some nonnegative integers 
$0 \leq m \leq n$, except for $d \in\{2,3,5,15,26\}$. Furthermore, they explicitly stated all the solutions for these exceptional cases.

In the same spirit of the main result of Luca et al. [17], Rihane et al. [21] studied the Diophantine equations

$$
x_{k}=P_{n} \quad \text { and } \quad X_{k}=P_{n},
$$

where $x_{k}$ and $X_{k}$ are given by (3) and (4), respectively. They proved that for each square-free integer $d \geq 2$, there is at most one positive integer $x$ participating in the Pell equation (1) and at most one positive integer $X$ participating in the Pell equation (2) that is a Padovan number with a few exceptions of $d$ that they effectively computed. Furthermore, the exceptional cases were $d \in\{2,3,5,6\}$ and $d=5$ for the the first and second equations in (7), respectively. Several other related problems have been studied where $x_{k}$ belongs to some interesting positive integer sequences. For example, see $[8,9,11,12,14-16,18]$.

\section{Main results}

In this paper, we study the same problem considered by E. F. Bravo et al.[3, 4] but with Padovan numbers instead of Tribonacci numbers. We also extend the results from the Pell equation (1) to the Pell equation (2). In both cases we find that there are only finitely many solutions that we effectively compute. Since $P_{1}=P_{2}=P_{3}=1$, we discard the situations when $n=1$ and $n=2$ and just count the solutions for $n=3$. Similarly, $P_{4}=P_{5}=2$, so we just count the solutions for $n=5$.

The main aim of this paper is to prove the following results.

Theorem 1 For each square-free integer $d \geq 2$, there is at most one positive integer $k$ such that

$$
x_{k}=P_{n}+P_{m}
$$

except when $d \in\{2,3,6,15,110,483\}$ in the +1 case and $d \in\{2,5,10,17\}$ in the -1 case.

Theorem 2 For each square-free integer $d \geq 2$, there is at most one positive integer $k$ such that

$$
X_{k}=P_{n}+P_{m}
$$

except when $d \in\{3,5,21\}$ in the +4 case and $d \in\{2,5\}$ in the -4 case.

For the exceptional values of $d$ listed in Theorem 1 and Theorem 2, all solutions $(k, n, m)$ are listed at the end of the proof of each result. The main tools used in this paper are lower bounds for nonzero linear forms in logarithms of algebraic numbers "á la Baker" and the Baker-Davenport reduction procedure, as well as the elementary properties of the Padovan sequence and solutions to Pell equations. Computations are done with the help of a computer program in Mathematica. 


\section{Preliminary results}

\subsection{The Padovan sequence}

Here, we recall some important properties of the Padovan sequence $\left(P_{n}\right)_{n \geq 0}$. The characteristic equation

$$
x^{3}-x-1=0,
$$

has roots $\alpha, \beta, \gamma=\bar{\beta}$, where

$$
\alpha=\frac{r_{1}+r_{2}}{6}, \quad \beta=\frac{-\left(r_{1}+r_{2}\right)+\sqrt{-3}\left(r_{1}-r_{2}\right)}{12},
$$

with

$$
r_{1}=\sqrt[3]{108+12 \sqrt{69}} \text { and } r_{2}=\sqrt[3]{108-12 \sqrt{69}}
$$

Furthermore, a Binet-like formula for Padovan numbers is given by

$$
P_{n}=a \alpha^{n}+b \beta^{n}+c \gamma^{n} \quad \text { for all } \quad n \geq 0,
$$

where

$$
a=\frac{\alpha+1}{(\alpha-\beta)(\alpha-\gamma)}, \quad b=\frac{\beta+1}{(\beta-\alpha)(\beta-\gamma)}, \quad c=\frac{\gamma+1}{(\gamma-\alpha)(\gamma-\beta)}=\bar{b} .
$$

Numerically, the following estimates hold:

$$
\begin{aligned}
& 1.32<\alpha<1.33 \\
& 0.86<|\beta|=|\gamma|=\alpha^{-\frac{1}{2}}<0.87 \\
& 0.54<a<0.55 \\
& 0.28<|b|=|c|<0.29
\end{aligned}
$$

From (10), (11), and (14), it is easy to see that the contribution of the complex conjugate roots $\beta$ and $\gamma$, to the right-hand side of (12), is very small. More exactly, setting $e(n):=P_{n}-a \alpha^{n}$ and taking into account the facts that $|\beta|=|\gamma|=\alpha^{-\frac{1}{2}}$ and $|b|=|c|<0.29$ (by (14)), it follows that, for any $n \geq 1$,

$$
|e(n)|=\left|b \beta^{n}+c \gamma^{n}\right| \leq|b||\beta|^{n}+|c||\gamma|^{n}=|b| \alpha^{-\frac{n}{2}}+|c| \alpha^{-\frac{n}{2}}<2 \cdot 0.29 \cdot \alpha^{-\frac{n}{2}}<\frac{1}{\alpha^{n / 2}} .
$$

Finally, one can prove by induction that

$$
\alpha^{n-2} \leq P_{n} \leq \alpha^{n-1} \quad \text { holds for all } \quad n \geq 4 .
$$




\subsection{Linear forms in logarithms}

Let $\eta$ be an algebraic number of degree $d$ with minimal primitive polynomial over the integers

$$
a_{0} x^{d}+a_{1} x^{d-1}+\cdots+a_{d}=a_{0} \prod_{i=1}^{d}\left(x-\eta^{(i)}\right),
$$

where the leading coefficient $a_{0}$ is positive and the $\eta^{(i)}$ 's are the conjugates of $\eta$. Then, the logarithmic height of $\eta$ is given by

$$
h(\eta):=\frac{1}{d}\left(\log a_{0}+\sum_{i=1}^{d} \log \left(\max \left\{\left|\eta^{(i)}\right|, 1\right\}\right)\right) .
$$

In particular, if $\eta=p / q$ is a rational number with $\operatorname{gcd}(p, q)=1$ and $q>0$, then $h(\eta)=\log \max \{|p|, q\}$. The following are some of the properties of the logarithmic height function $h(\cdot)$, which will be used in the next sections of this paper without reference:

$$
\begin{aligned}
h\left(\eta_{1} \pm \eta_{2}\right) & \leq h\left(\eta_{1}\right)+h\left(\eta_{2}\right)+\log 2, \\
h\left(\eta_{1} \eta_{2}^{ \pm 1}\right) & \leq h\left(\eta_{1}\right)+h\left(\eta_{2}\right), \\
h\left(\eta^{s}\right) & =|s| h(\eta) \quad(s \in \mathbb{Z}) .
\end{aligned}
$$

We recall the result of Bugeaud et al. (see [6], Theorem 9.4), which is a modified version of the result of Matveev [19], which is one of our main tools in this paper.

Theorem 3 (Matveev according to Bugeaud et al., $[6,19])$ Let $\eta_{1}, \ldots, \eta_{t}$ be nonzero elements of an algebraic number field $\mathbb{K} \subset \mathbb{R}$ of degree $D_{\mathbb{K}}$ over $\mathbb{Q}, b_{1}, \ldots, b_{t}$ be nonzero integers, and assume that

$$
\Lambda:=\eta_{1}^{b_{1}} \cdots \eta_{t}^{b_{t}}-1
$$

is nonzero. Then

$$
\log |\Lambda|>-1.4 \times 30^{t+3} \times t^{4.5} \times D_{\mathbb{K}}^{2}\left(1+\log D_{\mathbb{K}}\right)(1+\log B) A_{1} \cdots A_{t},
$$

where

$$
B \geq \max \left\{\left|b_{1}\right|, \ldots,\left|b_{t}\right|\right\}
$$

and

$$
A_{i} \geq \max \left\{D_{\llbracket} h\left(\eta_{i}\right),\left|\log \eta_{i}\right|, 0.16\right\}, \quad \text { for all } \quad i=1, \ldots, t .
$$




\subsection{Reduction procedure}

During the calculations, we get upper bounds on our variables which are too large, thus we need to reduce them. To do so, we use some results from the theory of continued fractions.

For the treatment of linear forms homogeneous in two integer variables, we use the following well-known classical result in the theory of Diophantine approximation. For further details, we refer the reader to the books of Baker and Wüstholz [2] and Cohen [7].

Lemma 1 (Legendre, $[2,7])$. Let $\tau$ be an irrational number, $p_{0} / q_{0}, p_{1} / q_{1}, p_{2} / q_{2}, \ldots$ be all the convergents of the continued fraction expansion of $\tau$ and $M$ be a positive integer. Let $N$ be a nonnegative integer such that $q_{N}>M$. Then putting $a(M):=\max \left\{a_{i}: i=0,1,2, \ldots, N\right\}$, the inequality

$$
\left|\tau-\frac{r}{s}\right|>\frac{1}{(a(M)+2) s^{2}},
$$

holds for all pairs $(r, s)$ of positive integers with $0<s<M$.

For a nonhomogeneous linear form in two integer variables, we use a slight variation of a result due to Dujella and Pethő (see [10], Lemma 5a) and itself is a generalization of a result of Baker and Davenport [1]. In this paper, we use an immediate variation of the result of Dujella and Pethő [10] due to J.J. Bravo et al. (see [5], Lemma 1). For a real number $X$, we write $\|X\|:=\min \{|X-n|: n \in \mathbb{Z}\}$ for the distance from $X$ to the nearest integer.

Lemma 2 (Dujella, Pethö according to J. J. Bravo et al. [5, 10]) Let $M$ be a positive integer, $p / q$ be a convergent of the continued fraction expansion of the irrational number $\tau$ such that $q>6 M$, and $A, B, \mu$ be some real numbers with $A>0$ and $B>1$. Furthermore, let $\varepsilon:=\|\mu q\|-M\|\tau q\|$. If $\varepsilon>0$, then there is no solution to the inequality

$$
0<|u \tau-v+\mu|<A B^{-w},
$$

in positive integers $u, v$, and $w$ with

$$
u \leq M \quad \text { and } \quad w \geq \frac{\log (A q / \varepsilon)}{\log B} .
$$

At various occasions, we need to find a lower bound for linear forms in logarithms with bounded integer coefficients in three and four variables. In this case, we use the LLL algorithm that we describe below. Let $\tau_{1}, \tau_{2}, \ldots \tau_{t} \in \mathbb{R}$ and the linear form

$$
x_{1} \tau_{1}+x_{2} \tau_{2}+\cdots+x_{t} \tau_{t} \quad \text { with } \quad\left|x_{i}\right| \leq X_{i} .
$$

We put $X:=\max \left\{X_{i}\right\}, C>(t X)^{t}$ and consider the integer lattice $\Omega$ generated by 


$$
\mathbf{b}_{j}:=\mathbf{e}_{j}+\left\lfloor C \tau_{j}\right\rceil \mathbf{e}_{t} \quad \text { for } \quad 1 \leq j \leq t-1 \quad \text { and } \quad \mathbf{b}_{t}:=\left\lfloor C \tau_{t}\right\rceil \mathbf{e}_{t},
$$

where $C$ is a sufficiently large positive constant.

Lemma 3 (LLL-algorithm, [7]) Let $X_{1}, X_{2}, \ldots, X_{t}$ be positive integers such that $X:=\max \left\{X_{i}\right\}$ and $C>(t X)^{t}$ be a fixed sufficiently large constant. With the above notation on the lattice $\Omega$, we consider a reduced base $\left\{\mathbf{b}_{i}\right\}$ to $\Omega$ and its associated Gram-Schmidt orthogonalization base $\left\{\mathbf{b}_{i}^{*}\right\}$. We set

$$
c_{1}:=\max _{1 \leq i \leq t} \frac{\left\|\mathbf{b}_{1}\right\|}{\left\|\mathbf{b}_{i}^{*}\right\|}, \quad \theta:=\frac{\left\|\mathbf{b}_{1}\right\|}{c_{1}}, \quad Q:=\sum_{i=1}^{t-1} X_{i}^{2}, \quad \text { and } \quad R:=\frac{1}{2}\left(1+\sum_{i=1}^{t} X_{i}\right) .
$$

If the integers $x_{i}$ are such that $\left|x_{i}\right| \leq X_{i}$, for $1 \leq i \leq t$ and $\theta^{2} \geq Q+R^{2}$, then we have

$$
\left|\sum_{i=1}^{t} x_{i} \tau_{i}\right| \geq \frac{\sqrt{\theta^{2}-Q}-R}{C}
$$

For the proof and further details, we refer the reader to the book of Cohen (see [7], Proposition 2.3.20).

Finally, the following Lemma is also useful. It is Lemma 7 in [13].

Lemma 4 (Gúzman Sánchez, Luca, [13]) Let $r, H$, and L be positive real numbers. If $r \geq 1, H>\left(4 r^{2}\right)^{r}$, and $H>L /(\log L)^{r}$, then

$$
L<2^{r} H(\log H)^{r} .
$$

\section{Proof of Theorem 1}

Let $\left(x_{1}, y_{1}\right)$ be the smallest positive integer solution to the Pell equation (1). We put

$$
\delta:=x_{1}+y_{1} \sqrt{d} \text { and } \sigma:=x_{1}-y_{1} \sqrt{d} .
$$

From which we get that

$$
\delta \cdot \sigma=x_{1}^{2}-d y_{1}^{2}=: N, \quad \text { where } \quad N \in\{ \pm 1\} .
$$

Then,

$$
x_{k}=\frac{1}{2}\left(\delta^{k}+\sigma^{k}\right)
$$

Since $\delta \geq 1+\sqrt{2}$, it follows that the estimate

$$
\frac{\delta^{k}}{\alpha^{4}} \leq x_{k} \leq \delta^{k} \quad \text { holds for all } \quad k \geq 1
$$

We assume that $\left(k_{1}, n_{1}, m_{1}\right)$ and $\left(k_{2}, n_{2}, m_{2}\right)$ are triples of integers such that 


$$
x_{k_{1}}=P_{n_{1}}+P_{m_{1}} \quad \text { and } \quad x_{k_{2}}=P_{n_{2}}+P_{m_{2}} .
$$

We assume that $1 \leq k_{1}<k_{2}$. We also assume that $3 \leq m_{i}<n_{i}$ for $i=1,2$. We set $(k, n, m):=\left(k_{i}, n_{i}, m_{i}\right)$, for $i=1,2$. Using the inequalities (16) and (20), we get from (21) that

$$
\frac{\delta^{k}}{\alpha^{4}} \leq x_{k}=P_{n}+P_{m} \leq 2 \alpha^{n-1} \quad \text { and } \quad \alpha^{n-2} \leq P_{n}+P_{m}=x_{k} \leq \delta^{k} .
$$

The above inequalities give

$$
(n-2) \log \alpha<k \log \delta<(n+3) \log \alpha+\log 2 .
$$

Dividing through by $\log \alpha$ and setting $c_{2}:=1 / \log \alpha$, we get that

$$
-2<c_{2} k \log \delta-n<3+c_{2} \log 2,
$$

and since $\alpha^{3}>2$, we get

$$
\left|n-c_{2} k \log \delta\right|<6 \text {. }
$$

Furthermore, $k<n$, for if not, we would then get that

$$
\delta^{n} \leq \delta^{k}<2 \alpha^{n+3}, \quad \text { implying }\left(\frac{\delta}{\alpha}\right)^{n}<2 \alpha^{3},
$$

which is false since $\delta \geq 1+\sqrt{2}, \alpha \in(1.32,1.33)$ (by (14)), and $n \geq 4$.

Besides, given that $k_{1}<k_{2}$, we have by (16) and (21) that

$$
\alpha^{n_{1}-2} \leq P_{n_{1}} \leq P_{n_{1}}+P_{m_{1}}=x_{k_{1}}<x_{k_{2}}=P_{n_{2}}+P_{m_{2}} \leq 2 P_{n_{2}}<2 \alpha^{n_{2}-1} .
$$

Thus, we get that

$$
n_{1}<n_{2}+4
$$

\subsection{An inequality for $n$ and $k$}

Using the Eqs. (8), (12), (19), and (21), we have

$$
\frac{1}{2}\left(\delta^{k}+\sigma^{k}\right)=P_{n}+P_{m}=a \alpha^{n}+e(n)+a \alpha^{m}+e(m) .
$$

Therefore,

$$
\frac{1}{2} \delta^{k}-a\left(\alpha^{n}+\alpha^{m}\right)=-\frac{1}{2} \sigma^{k}+e(n)+e(m),
$$

and by (15), we have 


$$
\begin{aligned}
\left|\delta^{k}(2 a)^{-1} \alpha^{-n}\left(1+\alpha^{m-n}\right)^{-1}-1\right| \leq & \frac{1}{2 \delta^{k} a\left(\alpha^{n}+\alpha^{m}\right)}+\frac{2|b|}{\alpha^{n / 2} a\left(\alpha^{n}+\alpha^{m}\right)} \\
& +\frac{2|b|}{\alpha^{m / 2} a\left(\alpha^{n}+\alpha^{m}\right)} \\
\leq & \frac{1}{a \alpha^{n}}\left(\frac{1}{2 \delta^{k}}+\frac{2|b|}{\alpha^{n / 2}}+\frac{2|b|}{\alpha^{m / 2}}\right)<\frac{1.5}{\alpha^{n}} .
\end{aligned}
$$

Thus, we have

$$
\left|\delta^{k}(2 a)^{-1} \alpha^{-n}\left(1+\alpha^{m-n}\right)^{-1}-1\right|<\frac{1.5}{\alpha^{n}} .
$$

Put

$$
\Lambda_{1}:=\delta^{k}(2 a)^{-1} \alpha^{-n}\left(1+\alpha^{m-n}\right)^{-1}-1
$$

and

$$
\Gamma_{1}:=k \log \delta-\log (2 a)-n \log \alpha-\log \left(1+\alpha^{m-n}\right) .
$$

Since $\left|\Lambda_{1}\right|=\left|e^{\Gamma_{1}}-1\right|<1 / 2$ for $n \geq 4$ (because $1.5 / \alpha^{4}<1 / 2$ ), and the inequality $|y|<2\left|e^{y}-1\right|$ holds for all $y \in(-1 / 2,1 / 2)$, it follows that $e^{\left|\Gamma_{1}\right|}<2$ and so

$$
\left|\Gamma_{1}\right|<e^{\left|\Gamma_{1}\right|}\left|e^{\Gamma_{1}}-1\right|<\frac{3}{\alpha^{n}} .
$$

Thus, we get that

$$
\left|k \log \delta-\log (2 a)-n \log \alpha-\log \left(1+\alpha^{m-n}\right)\right|<\frac{3}{\alpha^{n}} .
$$

We apply Theorem 3 on the left-hand side of (24) with the data:

$$
\begin{aligned}
t:=4, \quad \eta_{1}:=\delta, \quad \eta_{2}:=2 a, \quad \eta_{3}:=\alpha, \quad \eta_{4}:=1+\alpha^{m-n}, \\
b_{1}:=k, \quad b_{2}:=-1, \quad b_{3}:=-n, \quad b_{4}:=-1 .
\end{aligned}
$$

Furthermore, we take the number field $\mathbb{K}:=\mathbb{Q}(\sqrt{d}, \alpha)$ which has degree $D_{\mathbb{K}}:=6$. Since $\max \{1, k, n\} \leq n$, we take $B:=n$. First, we note that the left-hand side of (24) is nonzero, since otherwise,

$$
\delta^{k}=2 a\left(\alpha^{n}+\alpha^{m}\right) .
$$

The left-hand side belongs to the quadratic field $\mathbb{Q}(\sqrt{d})$ while the right-hand side belongs to the cubic field $\mathbb{Q}(\alpha)$. These fields only intersect when both sides are rational numbers. Since $\delta^{k}$ is a positive algebraic integer and a unit, we get that to $\delta^{k}=1$. Hence, $k=0$, which is a contradiction. Thus, $\Lambda_{1} \neq 0$ and we can apply Theorem 3.

We have $h\left(\eta_{1}\right)=h(\delta)=(\log \delta) / 2$ and $h\left(\eta_{3}\right)=h(\alpha)=(\log \alpha) / 3$. Furthermore, 


$$
2 a=\frac{2 \alpha(\alpha+1)}{2 \alpha+3}
$$

The minimal polynomial of $2 a$ is $23 x^{3}-20 x-8$ and has roots $2 a, 2 b, 2 c$. Since $2|b|=2|c|<1$ (by (14)), then

$$
h\left(\eta_{2}\right)=h(2 a)=\frac{1}{3}(\log 23+\log (2 a)) .
$$

On the other hand,

$$
\begin{aligned}
h\left(\eta_{4}\right) & =h\left(1+\alpha^{m-n}\right) \leq h(1)+h\left(\alpha^{m-n}\right)+\log 2 \\
& =(n-m) h(\alpha)+\log 2=\frac{1}{3}(n-m) \log \alpha+\log 2 .
\end{aligned}
$$

Thus, we can take $A_{1}:=3 \log \delta$,

$$
A_{2}:=2(\log 23+\log (2 a)), \quad A_{3}:=2 \log \alpha, \quad A_{4}:=2(n-m) \log \alpha+6 \log 2 .
$$

Now, Theorem 3 tells us that

$$
\begin{aligned}
\log \left|\Lambda_{1}\right|> & -1.4 \times 30^{7} \times 4^{4.5} \times 6^{2}(1+\log 6)(1+\log n)(3 \log \delta) \\
& \times(2(\log 23+\log (2 a))(2 \log \alpha)(2(n-m) \log \alpha+6 \log 2) \\
> & -2.33 \times 10^{17}(n-m)(\log n)(\log \delta) .
\end{aligned}
$$

Comparing the above inequality with (24), we get

$$
n \log \alpha-\log 1.5<2.33 \times 10^{17}(n-m)(\log n)(\log \delta) .
$$

Hence, we get that

$$
n<8.30 \times 10^{17}(n-m)(\log n)(\log \delta) .
$$

We now return to the Diophantine equation (8) and rewrite it as

$$
\frac{1}{2} \delta^{k}-a \alpha^{n}=-\frac{1}{2} \sigma^{k}+e(n)+P_{m},
$$

we obtain

$$
\left|\delta^{k}(2 a)^{-1} \alpha^{-n}-1\right| \leq \frac{1}{a \alpha^{n-m}}\left(\frac{1}{\alpha}+\frac{1}{\alpha^{m+n / 2}}+\frac{1}{2 \delta^{k} \alpha^{m}}\right)<\frac{2.5}{\alpha^{n-m}} .
$$

Put

$$
\Lambda_{2}:=\delta^{k}(2 a)^{-1} \alpha^{-n}-1, \quad \Gamma_{2}:=k \log \delta-\log (2 a)-n \log \alpha .
$$

We assume for technical reasons that $n-m \geq 10$. Therefore, $\left|e^{\Lambda_{2}}-1\right|<1 / 2$. It follows that 


$$
|k \log \delta-\log (2 a)-n \log \alpha|=\left|\Gamma_{2}\right|<e^{\left|\Lambda_{2}\right|}\left|e^{\Lambda_{2}}-1\right|<\frac{5}{\alpha^{n-m}} .
$$

Furthermore, $\Lambda_{2} \neq 0$ (so $\Gamma_{2} \neq 0$ ), since $\delta^{k} \notin \mathbb{Q}(\alpha)$ by a previous argument.

We now apply Theorem 3 to the left-hand side of (27) with the data

$$
t:=3, \quad \eta_{1}:=\delta, \quad \eta_{2}:=2 a, \quad \eta_{3}:=\alpha, \quad b_{1}:=k, \quad b_{2}:=-1, \quad b_{3}:=-n .
$$

Thus, we have the same $A_{1}, A_{2}, A_{3}$, as before. Then, by Theorem 3 , we conclude that

$$
\log |\Lambda|>-9.82 \times 10^{14}(\log \delta)(\log n)(\log \alpha) .
$$

By comparing with (27), we get

$$
n-m<9.84 \times 10^{14}(\log \delta)(\log n) .
$$

This was obtained under the assumption that $n-m \geq 10$, but if $n-m<10$, then the above inequality also holds as well. We replace $n-m$ in (26) by its upper bound that we obtained in (29) and use the fact that $\delta^{k} \leq 2 \alpha^{n+3}$, to obtain bounds on $n$ and $k$ in terms of $\log n$ and $\log \delta$. We now record what we have proved so far.

Lemma 5 Let $(k, n, m)$ be a solution to the Diophantine equation (8) with $3 \leq m<n$, then

$$
k<2.5 \times 10^{32}(\log n)^{2}(\log \delta) \text { and } n<8.2 \times 10^{32}(\log n)^{2}(\log \delta)^{2} .
$$

\subsection{Absolute bounds}

We recall that $(k, n, m)=\left(k_{i}, n_{i}, m_{i}\right)$, where $3 \leq m_{i}<n_{i}$, for $i=1,2$ and $1 \leq k_{1}<k_{2}$. Furthermore, $n_{i} \geq 4$ for $i=1,2$. We return to (28) and write

$$
\left|\Gamma_{2}^{(i)}\right|:=\left|k_{i} \log \delta-\log (2 a)-n_{i} \log \alpha\right|<\frac{5}{\alpha^{n_{i}-m_{i}}}, \quad \text { for } \quad i=1,2 .
$$

We do a suitable cross product between $\Gamma_{2}^{(1)}, \Gamma_{2}^{(2)}$ and $k_{1}, k_{2}$ to eliminate the term involving $\log \delta$ in the above linear forms in logarithms:

$$
\begin{aligned}
\left|\Gamma_{3}\right|: & =\left|\left(k_{1}-k_{2}\right) \log (2 a)+\left(k_{1} n_{2}-k_{2} n_{1}\right) \log \alpha\right|=\left|k_{2} \Gamma_{2}^{(1)}-k_{1} \Gamma_{2}^{(2)}\right| \\
& \leq k_{2}\left|\Gamma_{2}^{(1)}\right|+k_{1}\left|\Gamma_{2}^{(2)}\right| \leq \frac{5 k_{2}}{\alpha^{n_{1}-m_{1}}}+\frac{5 k_{1}}{\alpha^{n_{2}-m_{2}}} \leq \frac{10 n_{2}}{\alpha^{\lambda}},
\end{aligned}
$$

where $\lambda:=\min _{1 \leq i \leq 2}\left\{n_{i}-m_{i}\right\}$.

We need to find an upper bound for $\lambda$. If $10 n_{2} / \alpha^{\lambda}>1 / 2$, we then get

$$
\lambda<\frac{\log \left(20 n_{2}\right)}{\log \alpha}<4 \log \left(20 n_{2}\right) .
$$

Otherwise, $\left|\Gamma_{3}\right|<1 / 2$, so 


$$
\left|e^{\Gamma_{3}}-1\right|=\left|(2 a)^{k_{1}-k_{2}} \alpha^{k_{1} n_{2}-k_{2} n_{1}}-1\right|<2\left|\Gamma_{3}\right|<\frac{20 n_{2}}{\alpha^{\lambda}} .
$$

We apply Theorem 3 with the data: $t:=2, \eta_{1}:=2 a, \eta_{2}:=\alpha, b_{1}:=k_{1}-k_{2}$, $b_{2}:=k_{1} n_{2}-k_{2} n_{1}$. We take the number field $\mathbb{K}:=\mathbb{Q}(\alpha)$ and $D_{\mathbb{K}}:=3$. We begin by checking that $e^{\Gamma_{3}}-1 \neq 0$ (so $\Gamma_{3} \neq 0$ ). This is true, because $\alpha$ and $2 a$ are multiplicatively independent, since $\alpha$ is a unit in the ring of integers $\mathbb{Q}(\alpha)$ while the norm of $2 a$ is $8 / 23$.

We note that $\left|k_{1}-k_{2}\right|<k_{2}<n_{2}$. Furthermore, from (31), we have

$$
\left|k_{2} n_{1}-k_{1} n_{2}\right|<\left(k_{2}-k_{1}\right) \frac{|\log (2 a)|}{\log \alpha}+\frac{10 k_{2}}{\alpha^{\lambda} \log \alpha}<11 k_{2}<11 n_{2}
$$

given that $\lambda \geq 1$. Therefore, we can take $B:=11 n_{2}$. By Theorem 3 , with the same $A_{1}:=\log 23$ and $A_{2}:=\log \alpha$, we have that

$$
\log \left|e^{\Gamma_{3}}-1\right|>-1.55 \times 10^{11}\left(\log n_{2}\right)(\log \alpha) .
$$

By comparing this with (33), we get

$$
\lambda<1.56 \times 10^{11} \log n_{2} .
$$

Note that (34) is a better bound than (32), so (34) always holds. Without loss of generality, we can assume that $\lambda=n_{i}-m_{i}$, for $i=1,2$ fixed.

We set $\{i, j\}=\{1,2\}$ and return to $(25)$ to replace $(k, n, m)=\left(k_{i}, n_{i}, m_{i}\right)$ :

$$
\left|\Gamma_{1}^{(i)}\right|=\left|k_{i} \log \delta-\log (2 a)-n_{i} \log \alpha-\log \left(1+\alpha^{m_{i}-n_{i}}\right)\right|<\frac{3}{\alpha^{n_{i}}},
$$

and also return to $(28)$, with $(k, n, m)=\left(k_{j}, n_{j}, m_{j}\right)$ :

$$
\left|\Gamma_{2}^{(j)}\right|=\left|k_{j} \log \delta-\log (2 a)-n_{j} \log \alpha\right|<\frac{5}{\alpha^{n_{j}-m_{j}}} .
$$

We perform a cross product on (35) and (36) to eliminate the terms on $\log \delta$ :

$$
\begin{aligned}
\left|\Gamma_{4}\right|: & =\left|\left(k_{j}-k_{i}\right) \log (2 a)+\left(k_{j} n_{i}-k_{i} n_{j}\right) \log \alpha+k_{j} \log \left(1+\alpha^{m_{i}-n_{i}}\right)\right| \\
& =\left|k_{i} \Gamma_{2}^{(j)}-k_{j} \Gamma_{1}^{(i)}\right| \leq k_{i}\left|\Gamma_{2}^{(j)}\right|+k_{j}\left|\Gamma_{1}^{(i)}\right|<\frac{5 k_{i}}{\alpha^{n_{j}-m_{j}}}+\frac{3 k_{j}}{\alpha^{n_{i}}}<\frac{8 n_{2}}{\alpha^{v}}
\end{aligned}
$$

with $v:=\min \left\{n_{i}, n_{j}-m_{j}\right\}$. As before, we need to find an upper bound on $v$. If $8 n_{2} / \alpha^{v}>1 / 2$, then we get

$$
v<\frac{\log \left(16 n_{2}\right)}{\log \alpha}<4 \log \left(16 n_{2}\right) .
$$

Otherwise, $\left|\Gamma_{4}\right|<1 / 2$, so we have

$$
\left|e^{\Gamma_{4}}-1\right| \leq 2\left|\Gamma_{4}\right|<\frac{16 n_{2}}{\alpha^{v}} .
$$

To apply Theorem 3 , first if $e^{\Gamma_{4}}=1$, we obtain 


$$
(2 a)^{k_{i}-k_{j}}=\alpha^{k_{j} n_{i}-k_{i} n_{j}}\left(1+\alpha^{-\lambda}\right)^{k_{j}} .
$$

Since $\alpha$ is a unit, the right-hand side in above is an algebraic integer. This is a contradiction, because $k_{1}<k_{2}$ so $k_{i}-k_{j} \neq 0$, and neither $(2 a)$ nor $(2 a)^{-1}$ are algebraic integers. Hence, $e^{\Gamma_{4}} \neq 1$. By assuming that $v \geq 100$, we apply Theorem 3 with the data:

$$
\begin{gathered}
t:=3, \quad \eta_{1}:=2 a, \quad \eta_{2}:=\alpha, \quad \eta_{3}:=1+\alpha^{-\lambda}, \\
b_{1}:=k_{j}-k_{i}, \quad b_{2}:=k_{j} n_{i}-k_{i} n_{j}, \quad b_{3}:=k_{j},
\end{gathered}
$$

and the inequalities (34) and (39). We get

$$
v=\min \left\{n_{i}, n_{j}-m_{j}\right\}<1.14 \times 10^{14} \lambda \log n_{2}<1.78 \times 10^{25}\left(\log n_{2}\right)^{2} .
$$

The above inequality also holds when $v<100$. Furthermore, it also holds when the inequality (38) holds. Therefore, the above inequality holds in all cases. Note that the case $(i, j)=(2,1)$ leads to $n_{1}-m_{1} \leq n_{1} \leq n_{2}+4$ whereas $(i, j)=(1,2)$ leads to $v=\min \left\{n_{1}, n_{2}-m_{2}\right\}$. Hence, either the minimum is $n_{1}$, so

$$
n_{1}<1.78 \times 10^{25}\left(\log n_{2}\right)^{2},
$$

or the minimum is $n_{j}-m_{j}$ and from the inequality (34), we get that

$$
\max _{1 \leq j \leq 2}\left\{n_{j}-m_{j}\right\}<1.78 \times 10^{25}\left(\log n_{2}\right)^{2} .
$$

Next, we assume that we are in the case (41). We evaluate (35), for $i=1,2$ and make a suitable cross-product to eliminate the terms involving $\log \delta$ :

$$
\begin{aligned}
\left|\Gamma_{5}\right|:= & \mid\left(k_{2}-k_{1}\right) \log (2 a)+\left(k_{2} n_{1}-k_{1} n_{2}\right) \log \alpha \\
& +k_{2} \log \left(1+\alpha^{m_{1}-n_{1}}\right)-k_{1} \log \left(1+\alpha^{m_{2}-n_{2}}\right) \mid \\
= & \left|k_{1} \Gamma_{1}^{(2)}-k_{2} \Gamma_{1}^{(1)}\right| \leq k_{1}\left|\Gamma_{1}^{(2)}\right|+k_{2}\left|\Gamma_{1}^{(1)}\right|<\frac{6 n_{2}}{\alpha^{n_{1}}} .
\end{aligned}
$$

In the above inequality, we used the inequality (23) to conclude that $\min \left\{n_{1}, n_{2}\right\} \geq n_{1}-4$ as well as the fact that $n_{i} \geq 4$ for $i=1.2$. Next, we apply a linear form in four logarithms to obtain an upper bound to $n_{1}$. As in the previous calculations, we pass from (42) to

$$
\left|e^{\Gamma_{5}}-1\right|<\frac{12 n_{2}}{\alpha^{n_{1}}},
$$

which is implied by (42) except if $n_{1}$ is very small, say

$$
n_{1} \leq 4 \log \left(12 n_{2}\right) .
$$

Thus, we assume that (44) does not hold, therefore (43) holds. Then, to apply Theorem 3, we first justify that $e^{\Gamma_{5}} \neq 1$. Otherwise,

$$
(2 a)^{k_{1}-k_{2}}=\alpha^{k_{2} n_{1}-k_{1} n_{2}}\left(1+\alpha^{n_{1}-m_{1}}\right)^{k_{2}}\left(1+\alpha^{n_{2}-m_{2}}\right)^{-k_{1}} .
$$


By the fact that $k_{1}<k_{2}$, the norm $\mathbf{N}_{\mathbb{Q}(\alpha) / \mathbb{Q}}(2 a)=8 / 23$ and that $\alpha$ is a unit, we have that 23 divides the norm $\mathbf{N}_{\mathbb{K} / \mathbb{Q}}\left(1+\alpha^{n_{1}-m_{1}}\right)$. The factorization of the ideal generated by 23 in $\mathcal{O}_{\mathbb{Q}(\alpha)}$ is $(23)=\mathfrak{p}_{1}^{2} \mathfrak{p}_{2}$, where $\mathfrak{p}_{1}=(23, \alpha+13)$ and $\mathfrak{p}_{2}=(23, \alpha+20)$. Hence, $\mathfrak{p}_{2}$ divides $\alpha^{n_{1}-m_{1}}+1$. Given that $\alpha \equiv-20\left(\bmod \mathfrak{p}_{2}\right)$, then $(-20)^{n_{1}-m_{1}} \equiv-1\left(\bmod \mathfrak{p}_{2}\right)$. Taking the norm $\mathbf{N}_{\mathbb{Q}(\alpha) / \mathbb{Q}}$, we obtain that $(-20)^{n_{1}-m_{1}} \equiv-1(\bmod 23)$. If $n_{1}-m_{1}$ is even, -1 is a quadratic residue modulo 23 and if $n_{1}-m_{1}$ is odd then 20 is a quadratic residue modulo 23. But, neither -1 nor 20 are quadratic residues modulo 23 . Thus, $e^{\Gamma_{5}} \neq 1$.

Then, we apply Theorem 3 on the left-hand side of the inequality (43) with the data

$$
\begin{aligned}
t:=4, \quad \eta_{1}:=2 a, \quad \eta_{2}:=\alpha, \quad \eta_{3}:=1+\alpha^{m_{1}-n_{1}}, \quad \eta_{4}:=1+\alpha^{m_{2}-n_{2}}, \\
b_{1}:=k_{2}-k_{1}, \quad b_{2}:=k_{2} n_{1}-k_{1} n_{2}, \quad b_{3}:=k_{2}, \quad b_{4}:=k_{1} .
\end{aligned}
$$

Combining the right-hand side of (43) with the inequalities (34) and (41), Theorem 3 gives

$$
n_{1}<3.02 \times 10^{16}\left(n_{1}-m_{1}\right)\left(n_{2}-m_{2}\right)\left(\log n_{2}\right)<8.33 \times 10^{52}\left(\log n_{2}\right)^{4} .
$$

In the above, we used the facts that

$$
\min _{1 \leq i \leq 2}\left\{n_{i}-m_{i}\right\}<1.56 \times 10^{11} \log n_{2} \text { and } \max _{1 \leq i \leq 2}\left\{n_{i}-m_{i}\right\}<1.78 \times 10^{25}\left(\log n_{2}\right)^{2} .
$$

This was obtained under the assumption that the inequality (44) does not hold. If (44) holds, then so does (45). Thus, we have that inequality (45) holds provided that inequality (41) holds. Otherwise, inequality (40) holds which is a better bound than (45). Hence, we conclude that (45) holds in all possible cases.

By the inequality (22),

$$
\log \delta \leq k_{1} \log \delta \leq n_{1} \log \alpha+\log 6<2.38 \times 10^{52}\left(\log n_{2}\right)^{4} .
$$

By substituting this into (30) we get $n_{2}<4.64 \times 10^{137}\left(\log n_{2}\right)^{10}$, and then, by Lemma 4 , with the data $r:=10, H:=4.64 \times 10^{137}$ and $L:=n_{2}$, we get that $n_{2}<4.87 \times 10^{165}$. This immediately gives that $n_{1}<1.76 \times 10^{63}$.

We record what we have proved.

Lemma 6 Let $\left(k_{i}, n_{i}, m_{i}\right)$ be a solution to the Diophantine equation (8), with $3 \leq m_{i}<n_{i}$ for $i \in\{1,2\}$ and $1 \leq k_{1}<k_{2}$, then

$$
\max \left\{k_{1}, m_{1}\right\}<n_{1}<1.76 \times 10^{63}, \quad \text { and } \max \left\{k_{2}, m_{2}\right\}<n_{2}<4.87 \times 10^{165} \text {. }
$$




\section{Reducing the bounds for $n_{1}$ and $n_{2}$}

In this section, we reduce the bounds for $n_{1}$ and $n_{2}$ given in Lemma 6 to cases that can be computationally treated. For this, we return to the inequalities for $\Gamma_{3}, \Gamma_{4}$, and $\Gamma_{5}$.

\subsection{The first reduction}

We divide both sides of the inequality (31) by $\left(k_{2}-k_{1}\right) \log \alpha$. We get that

$$
\left|\frac{\log (2 a)}{\log \alpha}-\frac{k_{2} n_{1}-k_{1} n_{2}}{k_{2}-k_{1}}\right|<\frac{36 n_{2}}{\alpha^{\lambda}\left(k_{2}-k_{1}\right)} \quad \text { with } \quad \lambda:=\min _{1 \leq i \leq 2}\left\{n_{i}-m_{i}\right\}
$$

We assume that $\lambda \geq 10$. Below we apply Lemma 1 . We put $\tau:=\log (2 a) / \log \alpha$, which is irrational and compute its continued fraction

$$
\left[a_{0}, a_{1}, a_{2}, \ldots\right]=[1,3,3,1,11,1,2,1,1,1,3,1,1,1,2,5,1,15,2,19,1,1,2,2, \ldots],
$$

and its convergents

$$
\left[\frac{p_{0}}{q_{0}}, \frac{p_{1}}{q_{1}}, \frac{p_{2}}{q_{2}}, \ldots\right]=\left[1, \frac{4}{3}, \frac{13}{10}, \frac{17}{13}, \frac{200}{153}, \frac{217}{166}, \frac{634}{485}, \frac{851}{651}, \frac{1485}{1136}, \frac{2336}{1787}, \frac{8493}{6497}, \ldots\right] .
$$

Furthermore, we note that taking $M:=4.87 \times 10^{165}$ (by Lemma 6), it follows that

$$
q_{315}>M>n_{2}>k_{2}-k_{1} \quad \text { and } \quad a(M):=\max \left\{a_{i}: 0 \leq i \leq 315\right\}=a_{282}=2107 .
$$

Thus, by Lemma 1, we have that

$$
\left|\tau-\frac{k_{2} n_{1}-k_{1} n_{2}}{k_{2}-k_{1}}\right|>\frac{1}{2109\left(k_{2}-k_{1}\right)^{2}} .
$$

Hence, combining the inequalities (46) and (47), we obtain

$$
\alpha^{\lambda}<75924 n_{2}\left(k_{2}-k_{1}\right)<1.75 \times 10^{336},
$$

so $\lambda \leq 2714$. This was obtained under the assumption that $\lambda \geq 10$. Otherwise, $\lambda<10<2714$ holds as well.

Now, for each $n_{i}-m_{i}=\lambda \in[1,2714]$, we estimate a lower bound for $\left|\Gamma_{4}\right|$, with

$$
\Gamma_{4}=\left(k_{j}-k_{i}\right) \log (2 a)+\left(k_{j} n_{i}-k_{i} n_{j}\right) \log \alpha+k_{j} \log \left(1+\alpha^{m_{i}-n_{i}}\right)
$$

given in the inequality (37), via the procedure described in Sect. 3.3 (LLL-algorithm). We recall that $\Gamma_{4} \neq 0$. We apply Lemma 3 with the data:

$$
\begin{aligned}
t:=3, \quad \tau_{1}:=\log (2 a), \quad \tau_{2}:=\log \alpha, \quad \tau_{3}:=\log \left(1+\alpha^{-\lambda}\right), \\
x_{1}:=k_{j}-k_{i}, \quad x_{2}:=k_{j} n_{i}-k_{i} n_{j}, \quad x_{3}:=k_{j} .
\end{aligned}
$$

We set $X:=5.4 \times 10^{166}$ as an upper bound to $\left|x_{i}\right|<11 n_{2}$ for all $i=1,2,3$, and 
$C:=(20 X)^{5}$. A computer search using Mathematica allows us to conclude, together with the inequality (37), that

$$
2 \times 10^{-671}<\min _{1 \leq \lambda \leq 2714}\left|\Gamma_{4}\right|<8 n_{2} \alpha^{-v}, \quad \text { with } \quad v:=\min \left\{n_{i}, n_{j}-m_{j}\right\}
$$

which leads to $v \leq 6760$. As we have noted before, $v=n_{1}$ (so $n_{1} \leq 6760$ ) or $v=n_{j}-m_{j}$.

Next, we suppose that $n_{j}-m_{j}=v \leq 6760$. Since $\lambda \leq 2714$, we have

$$
\lambda:=\min _{1 \leq i \leq 2}\left\{n_{i}-m_{i}\right\} \leq 2714 \text { and } \chi:=\max _{1 \leq i \leq 2}\left\{n_{i}-m_{i}\right\} \leq 6760 .
$$

Now, returning to the inequality (42) which involves

$$
\begin{aligned}
\Gamma_{5}:= & \left(k_{2}-k_{1}\right) \log (2 a)+\left(k_{2} n_{1}-k_{1} n_{2}\right) \log \alpha \\
& +k_{2} \log \left(1+\alpha^{m_{1}-n_{1}}\right)-k_{1} \log \left(1+\alpha^{m_{2}-n_{2}}\right) \neq 0 .
\end{aligned}
$$

We use again the LLL algorithm to estimate the lower bound for $\left|\Gamma_{5}\right|$ and thus, find a bound for $n_{1}$ that is better than the one given in Lemma 6 .

We distinguish the cases $\lambda<\chi$ and $\lambda=\chi$.

\subsection{The case $\lambda<\chi$}

We take $\lambda \in[1,2714]$ and $\chi \in[\lambda+1,6760]$ and apply Lemma 3 with the data: $t:=4$,

$$
\begin{gathered}
\tau_{1}:=\log (2 a), \quad \tau_{2}:=\log \alpha, \quad \tau_{3}:=\log \left(1+\alpha^{m_{1}-n_{1}}\right), \quad \tau_{4}:=\log \left(1+\alpha^{m_{2}-n_{2}}\right), \\
x_{1}:=k_{2}-k_{1}, \quad x_{2}:=k_{2} n_{1}-k_{1} n_{2}, \quad x_{3}:=k_{2}, \quad x_{4}:=-k_{1} .
\end{gathered}
$$

We also put $X:=5.4 \times 10^{166}$ and $C:=(20 X)^{9}$. After a computer search in Mathematica together with the inequality (42), we can confirm that

$$
\begin{gathered}
8 \times 10^{-1342}<\min _{\substack{1 \leq \lambda \leq 2714 \\
\lambda+1 \leq \chi \leq 6760}}\left|\Gamma_{5}\right|<6 n_{2} \alpha^{-n_{1}} . \\
\end{gathered}
$$

This leads to the inequality

$$
\alpha^{n_{1}}<7.5 \times 10^{1341} n_{2} .
$$

Substituting for the bound $n_{2}$ given in Lemma 6, we get that $n_{1} \leq 12172$.

\subsection{The case $\lambda=\chi$}

In this case, we have

$$
\Lambda_{5}:=\left(k_{2}-k_{1}\right)\left(\log (2 a)+\log \left(1+\alpha^{m_{1}-n_{1}}\right)\right)+\left(k_{2} n_{1}-k_{1} n_{2}\right) \log \alpha \neq 0 .
$$

We divide through the inequality (42) by $\left(k_{2}-k_{1}\right) \log \alpha$ to obtain 


$$
\left|\frac{\log (2 a)+\log \left(1+\alpha^{m_{1}-n_{1}}\right)}{\log \alpha}-\frac{k_{2} n_{1}-k_{1} n_{2}}{k_{2}-k_{1}}\right|<\frac{21 n_{2}}{\alpha^{n_{1}}\left(k_{2}-k_{1}\right)} .
$$

We now put

$$
\tau_{\lambda}:=\frac{\log (2 a)+\log \left(1+\alpha^{-\lambda}\right)}{\log \alpha},
$$

and compute its continued fractions $\left[a_{0}^{(\lambda)}, a_{1}^{(\lambda)}, a_{2}^{(\lambda)}, \ldots\right]$, and its convergents $\left[p_{0}^{(\lambda)} / q_{0}^{(\lambda)}, p_{1}^{(\lambda)} / q_{1}^{(\lambda)}, p_{2}^{(\lambda)} / q_{2}^{(\lambda)}, \ldots\right]$, for each $\lambda \in[1,2714]$. Furthermore, for each case we find an integer $t_{\lambda}$ such that $q_{t_{\lambda}}^{(\lambda)}>M:=4.87 \times 10^{165}>n_{2}>k_{2}-k_{1}$ and calculate

$$
a(M):=\max _{1 \leq \lambda \leq 2714}\left\{a_{i}^{(\lambda)}: 0 \leq i \leq t_{\lambda}\right\} .
$$

A computer search in Mathematica reveals that for $\lambda=321, t_{\lambda}=330$ and $i=263$, we have that $a(M)=a_{321}^{(330)}=306269$. Hence, combining the conclusion of Lemma 1 and the inequality (50), we get

$$
\alpha^{n_{1}}<21 \times 306271 n_{2}\left(k_{2}-k_{1}\right)<1.525 \times 10^{338},
$$

so $n_{1} \leq 2730$. Hence, we obtain that $n_{1} \leq 12172$ holds in all cases $\left(v=n_{1}, \lambda<\chi\right.$ or $\lambda=\chi)$. By the inequality (22), we have that

$$
\log \delta \leq k_{1} \log \delta \leq n_{1} \log \alpha+\log 6<3475 .
$$

By considering the second inequality in (30), we can conclude that $n_{2} \leq 9.9 \times 10^{39}\left(\log n_{2}\right)^{2}$, which immediately yields $n_{2}<3.36 \times 10^{44}$, by a simple application of Lemma 4 . We summarise the first cycle of our reduction process as follows:

$$
n_{1} \leq 12172 \text { and } n_{2} \leq 3.36 \times 10^{44}
$$

From the above inequalities, we note that the upper bound on $n_{2}$ represents a very good reduction of the bound given in Lemma 6. Hence, we expect that if we restart our reduction cycle with the new bound on $n_{2}$, then we get a better bound on $n_{1}$. Thus, we return to the inequality (46) and take $M:=3.36 \times 10^{44}$. A computer search in Mathematica reveals that

$$
q_{88}>M>n_{2}>k_{2}-k_{1} \quad \text { and } \quad a(M):=\max \left\{a_{i}: 0 \leq i \leq 88\right\}=a_{54}=373,
$$

from which it follows that $\lambda \leq 752$. We now return to (48) and we put $X:=$ $3.36 \times 10^{44}$ and $C:=(10 X)^{5}$ and then apply the LLL algorithm in Lemma 3 to $\lambda \in[1,752]$. After a computer search, we get 


$$
5.33 \times 10^{-184}<\min _{1 \leq \lambda \leq 752}\left|\Gamma_{4}\right|<8 n_{2} \alpha^{-v}
$$

then $v \leq 1846$. By continuing under the assumption that $n_{j}-m_{j}=v \leq 1846$, we return to (49) and put $X:=3.36 \times 10^{44}, C:=(10 X)^{9}$ and $M:=3.36 \times 10^{44}$ for the case $\lambda<\chi$ and $\lambda=\chi$. After a computer search, we confirm that

$$
\begin{gathered}
2 \times 10^{-366}<\min _{1 \leq \lambda \leq 752}\left|\Gamma_{5}\right|<6 n_{2} \alpha^{-n_{1}}, \\
\lambda+1 \leq \chi \leq 1846
\end{gathered}
$$

gives $n_{1} \leq 3318$, and $a(M)=a_{175}^{(205)}=206961$, which leads to $n_{1} \leq 772$. Hence, in both cases $n_{1} \leq 3318$ holds. This gives $n_{2} \leq 5 \times 10^{42}$ by a similar procedure as before, and $k_{1} \leq 3125$.

We record what we have proved.

Lemma 7 Let $\left(k_{i}, n_{i}, m_{i}\right)$ be a solution to the Diophantine equation (8), with $3 \leq m_{i}<n_{i}$ for $i=1,2$ and $1 \leq k_{1}<k_{2}$, then

$$
m_{1}<n_{1} \leq 3318, \quad k_{1} \leq 3125, \quad \text { and } \quad n_{2} \leq 5 \times 10^{42} .
$$

\subsection{The final reduction}

Returning to (17) and (19) and using the fact that $\left(x_{1}, y_{1}\right)$ is the smallest positive solution to the Pell equation (1), we obtain

$$
\begin{aligned}
x_{k} & =\frac{1}{2}\left(\delta^{k}+\sigma^{k}\right)=\frac{1}{2}\left(\left(x_{1}+y_{1} \sqrt{d}\right)^{k}+\left(x_{1}-y_{1} \sqrt{d}\right)^{k}\right) \\
& =\frac{1}{2}\left(\left(x_{1}+\sqrt{x_{1}^{2} \mp 1}\right)^{k}+\left(x_{1}-\sqrt{x_{1}^{2} \mp 1}\right)^{k}\right):=Q_{k}^{ \pm}\left(x_{1}\right) .
\end{aligned}
$$

Thus, we return to the Diophantine equation $x_{k_{1}}=P_{n_{1}}+P_{m_{1}}$ and consider the equations

$$
Q_{k_{1}}^{+}\left(x_{1}\right)=P_{n_{1}}+P_{m_{1}} \quad \text { and } \quad Q_{k_{1}}^{-}\left(x_{1}\right)=P_{n_{1}}+P_{m_{1}},
$$

with $k_{1} \in[1,3125], m_{1} \in[3,3318]$ and $n_{1} \in\left[m_{1}+1,3318\right]$.

Besides the trivial case $k_{1}=1$, with the help of a computer search in Mathematica on the above equations in (51), we list the only nontrivial solutions in Table 1 . We also note that $3+2 \sqrt{2}=(1+\sqrt{2})^{2}$, so these solutions come from the same Pell equation when $d=2$.

From Table 1, we set each $\delta:=\delta_{t}$ for $t=1,2, \ldots, 17$. We then work on the linear forms in logarithms $\Gamma_{1}$ and $\Gamma_{2}$, to reduce the bound on $n_{2}$ given in Lemma 7 . From the inequality (28), for $(k, n, m):=\left(k_{2}, n_{2}, m_{2}\right)$, we write 
Table 1 Solutions to $Q_{k_{1}}^{ \pm}\left(x_{1}\right)=P_{n_{1}}+P_{m_{1}}$

\begin{tabular}{|c|c|c|c|c|}
\hline \multicolumn{5}{|c|}{$\overline{Q_{k_{1}}^{+}\left(x_{1}\right)}$} \\
\hline$k_{1}$ & $x_{1}$ & $y_{1}$ & $d$ & $\delta$ \\
\hline 2 & 2 & 1 & 3 & $2+\sqrt{3}$ \\
\hline 2 & 3 & 2 & 2 & $3+2 \sqrt{2}$ \\
\hline 2 & 4 & 1 & 15 & $4+\sqrt{15}$ \\
\hline 2 & 5 & 2 & 6 & $5+2 \sqrt{6}$ \\
\hline 2 & 21 & 2 & 110 & $21+2 \sqrt{110}$ \\
\hline 2 & 22 & 1 & 483 & $22+\sqrt{483}$ \\
\hline 2 & 47 & 4 & 138 & $47+4 \sqrt{138}$ \\
\hline \multicolumn{5}{|c|}{$\overline{Q_{k_{1}}^{-}\left(x_{1}\right)}$} \\
\hline$k_{1}$ & $x_{1}$ & $y_{1}$ & $d$ & $\delta$ \\
\hline 2 & 1 & 1 & 2 & $1+\sqrt{2}$ \\
\hline 2 & 2 & 1 & 5 & $2+\sqrt{5}$ \\
\hline 2 & 3 & 1 & 10 & $3+\sqrt{10}$ \\
\hline 2 & 4 & 1 & 17 & $4+\sqrt{17}$ \\
\hline 2 & 5 & 1 & 26 & $5+\sqrt{26}$ \\
\hline 2 & 9 & 1 & 82 & $9+\sqrt{82}$ \\
\hline 2 & 10 & 1 & 101 & $10+\sqrt{101}$ \\
\hline 2 & 17 & 1 & 290 & $17+\sqrt{290}$ \\
\hline 2 & 42 & 1 & 1765 & $42+\sqrt{1765}$ \\
\hline 2 & 47 & 1 & 2210 & $47+\sqrt{2210}$ \\
\hline 2 & 63 & 1 & 3970 & $63+\sqrt{3970}$ \\
\hline
\end{tabular}

$$
\left|k_{2} \frac{\log \delta_{t}}{\log \alpha}-n_{2}+\frac{\log (2 a)}{\log \left(\alpha^{-1}\right)}\right|<\left(\frac{5}{\log \alpha}\right) \alpha^{-\left(n_{2}-m_{2}\right)}, \quad \text { for } \quad t=1,2, \ldots, 17 .
$$

We put

$$
\tau_{t}:=\frac{\log \delta_{t}}{\log \alpha}, \quad \mu_{t}:=\frac{\log (2 a)}{\log \left(\alpha^{-1}\right)} \quad \text { and } \quad\left(A_{t}, B_{t}\right):=\left(\frac{5}{\log \alpha}, \alpha\right) .
$$

We note that $\tau_{t}$ is transcendental by Gelfond-Schneider Theorem (see [2], Theorem 2.1). Thus, $\tau_{t}$ is irrational. We can rewrite the inequality (52) as

$$
0<\left|k_{2} \tau_{t}-n_{2}+\mu_{t}\right|<A_{t} B_{t}^{-\left(n_{2}-m_{2}\right)}, \quad \text { for } \quad t=1,2, \ldots, 17
$$

We take $M:=5 \times 10^{42}$ which is the upper bound on $n_{2}$ according to Lemma 7 and apply Lemma 2 to the inequality (53). As before, for each $\tau_{t}$ with $t=1,2, \ldots, 17$, we compute its continued fraction $\left[a_{0}^{(t)}, a_{1}^{(t)}, a_{2}^{(t)}, \ldots\right]$ and its convergents 
$p_{0}^{(t)} / q_{0}^{(t)}, p_{1}^{(t)} / q_{1}^{(t)}, p_{2}^{(t)} / q_{2}^{(t)}, \ldots$. For each case, by means of a computer search in Mathematica, we find an integer $s_{t}$ such that

$$
q_{s_{t}}^{(t)}>3 \times 10^{43}=6 M \quad \text { and } \quad \epsilon_{t}:=\left\|\mu_{t} q^{(t)}\right\|-M\left\|\tau_{t} q^{(t)}\right\|>0 .
$$

We finally compute all the values of $b_{t}:=\left\lfloor\log \left(A_{t} q_{s_{t}}^{(t)} / \epsilon_{t}\right) / \log B_{t}\right\rfloor$. The values of $b_{t}$ correspond to the upper bounds on $n_{2}-m_{2}$, for each $t=1,2, \ldots, 17$, according to Lemma 2. The results of the computation for each $t$ are recorded in Table 2.

By replacing $(k, n, m):=\left(k_{2}, n_{2}, m_{2}\right)$ in the inequality (25), we can write

$$
\left|k_{2} \frac{\log \delta_{t}}{\log \alpha}-n_{2}+\frac{\log \left(2 a\left(1+\alpha^{-\left(n_{2}-m_{2}\right)}\right)\right)}{\log \left(\alpha^{-1}\right)}\right|<\left(\frac{3}{\log \alpha}\right) \alpha^{-n_{2}}, \quad \text { for } \quad t=1,2, \ldots, 17 \text {. }
$$

We now put

$$
\tau_{t}:=\frac{\log \delta_{t}}{\log \alpha}, \quad \mu_{t, n_{2}-m_{2}}:=\frac{\log \left(2 a\left(1+\alpha^{-\left(n_{2}-m_{2}\right)}\right)\right)}{\log \left(\alpha^{-1}\right)} \quad \text { and } \quad\left(A_{t}, B_{t}\right):=\left(\frac{3}{\log \alpha}, \alpha\right) .
$$

With the above notations, we can rewrite the inequality (54) as

$$
0<\left|k_{2} \tau_{t}-n_{2}+\mu_{t, n_{2}-m_{2}}\right|<A_{t} B_{t}^{-n_{2}}, \quad \text { for } t=1,2, \ldots, 17 .
$$

We again apply Lemma 2 to the inequality (55), for

Table 2 First reduction computation results

\begin{tabular}{llllll}
\hline$t$ & $\delta_{t}$ & $s_{t}$ & $q_{s_{t}}$ & $\epsilon_{t}>$ & $b_{t}$ \\
\hline 1 & $2+\sqrt{3}$ & 85 & $8.93366 \times 10^{43}$ & 0.3100 & 374 \\
2 & $4+\sqrt{15}$ & 90 & $3.90052 \times 10^{43}$ & 0.3124 & 371 \\
3 & $5+2 \sqrt{6}$ & 80 & $3.16032 \times 10^{43}$ & 0.0122 & 382 \\
4 & $21+2 \sqrt{110}$ & 88 & $6.33080 \times 10^{43}$ & 0.2200 & 374 \\
5 & $22+\sqrt{483}$ & 75 & $4.19689 \times 10^{43}$ & 0.2361 & 372 \\
6 & $47+4 \sqrt{138}$ & 96 & $7.76442 \times 10^{43}$ & 0.3732 & 373 \\
7 & $1+\sqrt{2}$ & 78 & $1.46195 \times 10^{44}$ & 0.3328 & 375 \\
8 & $2+\sqrt{5}$ & 94 & $1.48837 \times 10^{44}$ & 0.2146 & 377 \\
9 & $3+\sqrt{10}$ & 88 & $4.21425 \times 10^{43}$ & 0.1347 & 374 \\
10 & $4+\sqrt{17}$ & 92 & $1.11753 \times 10^{44}$ & 0.2529 & 375 \\
11 & $5+\sqrt{26}$ & 98 & $3.23107 \times 10^{43}$ & 0.1043 & 374 \\
12 & $9+\sqrt{82}$ & 74 & $5.25207 \times 10^{43}$ & 0.2181 & 373 \\
13 & $10+\sqrt{101}$ & 94 & $1.86122 \times 10^{44}$ & 0.2672 & 377 \\
14 & $17+\sqrt{290}$ & 87 & $1.06422 \times 10^{44}$ & 0.0193 & 384 \\
15 & $42+\sqrt{1765}$ & 78 & $3.81406 \times 10^{43}$ & 0.1768 & 373 \\
16 & $47+\sqrt{2210}$ & 94 & $3.92482 \times 10^{43}$ & 0.4476 & 370 \\
17 & $63+\sqrt{3970}$ & 85 & $6.00550 \times 10^{43}$ & 0.4056 & 371 \\
\hline & & & & &
\end{tabular}

Birkhäuser 


$$
t=1,2, \ldots, 17, \quad n_{2}-m_{2}=1,2, \ldots, b_{t}, \quad \text { with } \quad M:=5 \times 10^{43} .
$$

We take

$$
\epsilon_{t, n_{2}-m_{2}}:=\left\|\mu_{t} q^{\left(t, n_{2}-m_{2}\right)}\right\|-M\left\|\tau_{t} q^{\left(t, n_{2}-m_{2}\right)}\right\|>0
$$

and

$$
b_{t}=b_{t, n_{2}-m_{2}}:=\left\lfloor\log \left(A_{t} q_{s_{t}}^{\left(t, n_{2}-m_{2}\right)} / \epsilon_{t, n_{2}-m_{2}}\right) / \log B_{t}\right\rfloor .
$$

With the help of Mathematica, we obtain the results in Table 3.

Thus, $\max \left\{b_{t, n_{2}-m_{2}}: t=1,2, \ldots, 17\right.$ and $\left.n_{2}-m_{2}=1,2, \ldots b_{t}\right\} \leq 408$. So, by Lemma 2, we have that $n_{2} \leq 408$, for all $t=1,2, \ldots, 17$, and by the inequality (23) we have that $n_{1} \leq n_{2}+4$. From the fact that $\delta^{k} \leq 2 \alpha^{n+3}$, we can conclude that $k_{1}<k_{2} \leq 133$. Collecting everything together, our problem is reduced to search for the solutions for (21) in the following range

$$
1 \leq k_{1}<k_{2} \leq 133, \quad 0 \leq m_{1}<n_{1} \in[3,408], \quad \text { and } \quad 0 \leq m_{2}<n_{2} \in[3,408] .
$$

After a computer search for the solutions to the Diophantine equations in (21) on the range above, we obtained the following solutions, which are the only solutions for the exceptional $d$ cases we have stated in Theorem 1:

For the +1 case:

$$
\begin{aligned}
(d=2) x_{1} & =3=P_{6}+P_{0}=P_{5}+P_{3}, \quad x_{2}=17=P_{12}+P_{3} ; \\
(d=3) x_{1} & =2=P_{3}+P_{0}=P_{3}+P_{3}, x_{2}=7=P_{9}+P_{0}=P_{7}+P_{6}, \\
x_{3} & =26=P_{13}+P_{8} ; \\
(d=6) x_{1} & =5=P_{8}+P_{0}=P_{7}+P_{3}=P_{6}+P_{5}, \\
x_{2} & =49=P_{16}+P_{0}=P_{15}+P_{12}=P_{14}+P_{13} ; \\
(d=15) x_{1} & =4=P_{7}+P_{0}=P_{6}+P_{3}=P_{5}+P_{5}, \quad x_{2}=31=P_{14}+P_{6} ; \\
(d=110) x_{1} & =21=P_{13}+P_{0}=P_{12}+P_{8}=P_{11}+P_{10}, \\
x_{2} & =881=P_{26}+P_{17}=P_{25}+P_{22} ; \\
(d=483) x_{1} & =22=P_{13}+P_{3}, \quad x_{2}=967=P_{26}+P_{20}=P_{25}+P_{23} .
\end{aligned}
$$

For the -1 case:

Table 3 Final reduction computation results

\begin{tabular}{llllllllll}
\hline$t$ & 1 & 2 & 3 & 4 & 5 & 6 & 7 & 8 & 9 \\
\hline$b_{t, n_{2}-m_{2}}$ & 388 & 389 & 394 & 394 & 393 & 394 & 396 & 392 & 392 \\
\hline$t$ & 10 & 11 & 12 & 13 & 14 & 15 & 16 & 17 \\
\hline$b_{t, n_{2}-m_{2}}$ & 396 & 392 & 408 & 390 & 396 & 396 & 388 & 389 \\
\hline
\end{tabular}




$$
\begin{gathered}
(d=2) x_{1}=1=P_{3}+P_{0}, \quad x_{2}=7=P_{9}+P_{0}=P_{8}+P_{5}=P_{7}+P_{6}, \\
x_{3}=41=P_{15}+P_{7}=P_{14}+P_{10}=P_{13}+P_{12} \\
(d=5) x_{1}=2=P_{5}+P_{0}=P_{3}+P_{3}, \quad x_{2}=38=P_{15}+P_{3} \\
(d=10) x_{1}=3=P_{6}+P_{0}=P_{5}+P_{3}, \quad x_{2}=117=P_{19}+P_{6} \\
(d=17) x_{1}=4=P_{7}+P_{0}=P_{6}+P_{3}=P_{5}+P_{5}, \quad x_{2}=P_{22}+P_{6} .
\end{gathered}
$$

This completes the proof of Theorem 1 .

\section{Proof of Theorem 2}

The proof of Theorem 2 follows from similar steps, techniques, and arguments as given in the proof of Theorem 1. So, we do not give the details here. Below, we give the solutions to the Diophantine equation (9) for the exceptional $d$ cases stated in Theorem 2.

For the +4 case:

$$
\begin{aligned}
(d=3) X_{1} & =4=P_{7}+P_{0}=P_{6}+P_{3}=P_{5}+P_{5}, \\
X_{2} & =14=P_{11}+P_{5}=P_{10}+P_{8}, \quad X_{3}=52=P_{16}+P_{6} ; \\
(d=5) X_{1} & =3=P_{6}+P_{0}=P_{5}+P_{3}, \quad X_{2}=7=P_{9}+P_{0}=P_{7}+P_{6}, \\
X_{3} & =18=P_{12}+P_{5} ; \\
(d=21) X_{1} & =5=P_{8}+P_{0}=P_{7}+P_{3}=P_{6}+P_{5}, \\
X_{2} & =23=P_{13}+P_{5}=P_{12}+P_{9}, \quad X_{3}=2525=P_{30}+P_{11} .
\end{aligned}
$$

For the -4 case:

$$
\begin{gathered}
(d=2) X_{1}=2=P_{5}+P_{0}=P_{3}+P_{3}, \quad X_{2}=14=P_{11}+P_{5}=P_{10}+P_{8} \\
(d=5) X_{1}=1=P_{3}+P_{0}, \quad X_{2}=4=P_{7}+P_{0}=P_{6}+P_{3}=P_{5}+P_{5}, \\
X_{3}=11=P_{10}+P_{5}=P_{9}+P_{7}, \quad X_{4}=29=P_{14}+P_{3} .
\end{gathered}
$$

Acknowledgements The author thanks the anonymous referees and the editor for the careful reading of the manuscript and the useful comments and suggestions that greatly improved the quality of presentation of this paper. Part of the work in this paper was done when the author visited the Institut de Mathématiques de Bordeaux, Université de Bordeaux, in May 2019. He thanks this institution for its hospitality and the fruitful working environment.

Funding Open Access funding provided by Austrian Science Fund (FWF).

\section{Compliance with ethical standards}

Conflict of interest The authors declare that they do not have conflict of interests.

Ethical standard This research complies with ethical standards.

Open Access This article is licensed under a Creative Commons Attribution 4.0 International License, which permits use, sharing, adaptation, distribution and reproduction in any medium or format, as long as you give appropriate credit to the original author(s) and the source, provide a link to the Creative 
Commons licence, and indicate if changes were made. The images or other third party material in this article are included in the article's Creative Commons licence, unless indicated otherwise in a credit line to the material. If material is not included in the article's Creative Commons licence and your intended use is not permitted by statutory regulation or exceeds the permitted use, you will need to obtain permission directly from the copyright holder. To view a copy of this licence, visit http:// creativecommons.org/licenses/by/4.0/.

\section{References}

1. Baker, A., Davenport, H.: The equations $3 x^{2}-2=y^{2}$ and $8 x^{2}-7=z^{2}$. Q. J. Math. Oxford Ser. (2) 20(1), 129-137 (1969)

2. Baker, A., Wüstholz, G.: Logarithmic Forms and Diophantine Geometry. Cambridge University Press, Cambridge (2008)

3. Bravo, E.F., Gómez, C.A., Luca, F.: $X$-coordinates of Pell equations as sums of two tribonacci numbers. Period. Math. Hungar. 77(2), 175-190 (2018)

4. Bravo, E.F., Gómez, C.A., Luca, F.: Correction to: $X$-coordinates of Pell equations as sums of two tribonacci numbers. Period. Math. Hungar. 80(1), 145-146 (2020)

5. Bravo, J.J., Gómez, C.A., Luca, F.: Powers of two as sums of two $k$-Fibonacci numbers. Miskolc Math. Notes 17(1), 85-100 (2016)

6. Bugeaud, Y., Mignotte, M., Siksek, S.: Classical Classical and modular approaches to exponential Diophantine equations I. Fibonacci and Lucas perfect powers. Ann. Math. (2) 163(2), 969-1018 (2006)

7. Cohen, H.: Number Theory. Volume I: Tools and Diophantine Equations, Graduate Texts in Mathematics 239. Springer, Berlin (2007)

8. Ddamulira, M., Luca, F.: On the $x$-coordinates of Pell equations which are $k$-generalized Fibonacci numbers. J. Numb. Theory 207, 156-195 (2020)

9. Dossavi-Yovo, A., Luca, F., Togbé, A.: On the $X$-coordinates of Pell equations which are rep-digits. Publ. Math. Debrecen 88, 381-399 (2016)

10. Dujella, A., Pethő, A.: A generalization of a theorem of Baker and Davenport. Q. J. Math. Oxford Ser. (2) 49(195), 291-306 (1998)

11. Faye, B., Luca, F.: On the $X$-coordinates of Pell equations which are repdigits. Fibonacci Q. 56(1), 52-62 (2018)

12. Gómez, C.A., Luca, F.: Zeckendorf representations with at most two terms to $x$-coordinates of Pell equations. Sci. China Math. 64(4), 627-642 (2020)

13. Gúzman Sánchez, S., Luca, F.: Linear combinations of factorials and $s$-units in a binary recurrence sequence. Ann. Math. Qué. 38(2), 169-188 (2014)

14. Kafle, B., Luca, F., Togbé, A.: On the $x$-coordinates of Pell equations which are Fibonacci numbers II. Colloq. Math. 149(1), 75-85 (2017)

15. Kafle, B., Luca, F., Togbé, A.: $x$-Coordinates of Pell equations which are Tribonacci numbers II. Period. Math. Hungar. 79(2), 157-167 (2019)

16. Kafle, B., Luca, F., Togbé, A.: $X$-coordinates of Pell equations which are Lucas numbers. Bol. Soc. Mat. Mex. (3) 25(3), 481-493 (2019)

17. Luca, F., Montejano, A., Szalay, L., Togbé, A.: On the $x$-coordinates of Pell equations which are Tribonacci numbers. Acta Arith. 179(1), 25-35 (2017)

18. Luca, F., Togbé, A.: On the $x$-coordinates of Pell equations which are Fibonacci numbers. Math. Scand. 122(1), 18-30 (2018)

19. Matveev, E.M.: An explicit lower bound for a homogeneous rational linear form in the logarithms of algebraic numbers II, Izv. Ross. Akad. Nauk Ser. Mat. 64 (6) (2000), 125-180, In Russian. English translation in Izv. Math. 64 (6) (2000), 1217-1269

20. OEIS Foundation Inc. The On-Line Encyclopedia of Integer Sequences (2020). https://oeis.org/

21. Rihane, S.S., Hernane, M.O., Togbé, A.: The $x$-coordinates of Pell equations and Padovan numbers. Turk. J. Math. 43(1), 207-223 (2019)

Publisher's Note Springer Nature remains neutral with regard to jurisdictional claims in published maps and institutional affiliations. 